\title{
Emoticons convey emotion in CMC
}

\author{
Wonmi Ahn \\ Yonsei Univ. Dept of Psychology \\ Yonsei Univ., Sinchon-dong, \\ Seodaemun-gu, Seoul, Korea \\ Jeeyea Park \\ Yonsei Univ. Dept of Psychology \\ Yonsei Univ., Sinchon-dong, \\ Seodaemun-gu, Seoul, Korea \\ jeeyea86@naver.com \\ Kwang-hee Han \\ Yonsei Univ. Dept of Psychology \\ Yonsei Univ., Sinchon-dong, \\ Seodaemun-gu, Seoul, Korea \\ khan@yonsei.ac.kr
}

\begin{abstract}
Expressing emotions in Computer-mediated communication is less social because of the lack of nonverbal cues unlike regular face-to-face communication. The use of emoticons or icons for visualizing emotions may be employed to replicate nonverbal facial expressions in CMC. This present research examined to what extent the use of emoticons (emotion icons) depends on social context (2:task-oriented vs. socio-emotional) and emotional valence (3:positive vs. neutral vs. negative). Participants were asked to type freely messages on mobile prototype with emoticons or without for six different scenarios. Results showed that there is a trend of kind of context. That is, participants used more emoticons in task-oriented than in socio-emotional social contexts. Furthermore, subjects reacted significantly more often with an emoticon in positive valence than in neutral or negative valence. An interaction was found between valence and kind of context. These results seemed in line with the social norms in face-to-face communication. We present implications with a empirical experiment that the use of emoticons in social interaction on the mobile.
\end{abstract}

Emotion, Emotional Sign, Social Context, valence, CMC.

\section{INTRODUCTION}

Rezabek and Cochenour defined emotional icons (emoticons) as "visual cues formed from ordinary typographical symbols that when read sideways represent feelings or emotions (Rezabek \& Cochenour 1998)." Early research argued that text based forms of computer mediated communications lose nonverbal cues such as facial expressions, gestures of tone of conversation (Daft and Lengel, 1984). However, recent research argued that using emotional icons (emoticons) could express nonverbal cues missing in CMC (Walther 1992).

The results of the only experimental study (Derks et al. 2003) suggested that the contexts of communication affect emoticon use, but they tested the effect of contexts in short simulated paper-and pencil chat sessions. This study investigated the frequency of emoticon use in CMC depends on social context and emotional valence on text message based on mobile prototype. Ahn (2010) showed the frequency of emoticon use in gender imbalance. The gender ratio in this study is approximately balanced.

Research Hypothesis:

H1:In socio-emotional subjects more use emoticons with message than in task-oriented social context.
H2: Subjects use more often with an emoticon in positive valence than in neutral or negative valence. H3: It expects that there is an interaction between kind of context and valence.

\section{METHOD}

\subsection{Participants}

Twenty-seven university students participated in the experiment. They consisted of $17 \mathrm{females}$ and 10males $(M=20, S D=1.69)$.

\subsection{Procedure}

The mobile phone was displayed on the left side of the screen and instructions with scenarios appeared on the right side: Participants were asked to enter text message with emoticon or without individually. Each trial ended with participants pressing the "SEND" button. After completing all condition, participants showed their text message and completed an open-ended statement on reason why using emoticon or not.

\subsection{Independent variables}

Eighteen scenarios were presented to participants. Scenarios were generated by researchers and 
approved its suitability for this experiment in a pilot study. The scenarios varied in the kind of social context (2: task-oriented vs. socio-emotional) and in the valence (3: positive vs. neutral vs. negative). Thus, 2 kind of social context $x 3$ valence of social context within-subject design was used.

\subsection{Dependent variables}

Dependent variables of this study were the frequency of emoticons. Participants were free to use an emoticon from a list of ten selected emoticons. These emoticons (smiling, pleased, hopeless, crying, and surprised: there are 2 different versions each of them) is most common used in Korea [5]. Korean emoticons composed of a double-byte character set unlike Western emoticons.

\section{RESULTS}

A $2 \times 3$ repeated ANOVA was conducted to investigate the effects of kind of context and valence. There was a trend of kind of social context $(p=.086)$. Participant used emoticon more often in task-oriented $(M=1.96)$ than in socio-emotional social contexts $(M=1.56)$. This trend is a opposite effect to previous research (H1 NOT supported).

There was main effect of valence, $F(2,52)=8.643$, $p<.001, \eta_{p} 2=.249$. In positive context participants used significantly more emoticons $(M=2.28)$ than other valence (neutral, $M=1.43$; negative, $M=$ 1.57) [Figure 2] ( $\mathrm{H} 2$ supported).

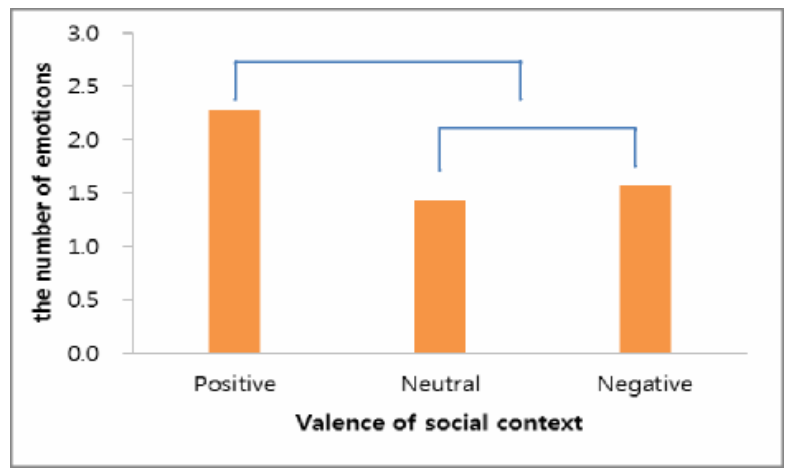

Figure 2: A main effect of Valence

Results showed a significant interaction between kind of social context and valence of social context $F(2,52)=35.922, p<.001, \eta p^{2}=.580$. In sadness, socio-emotional contexts participants used more emoticons $(M=2.19)$ than task-oriented context $(M=0.96)$. However in other valence of social contexts, emoticons were more used at task- oriented context than socio-emotional context [Figure 3] (H3 supported).

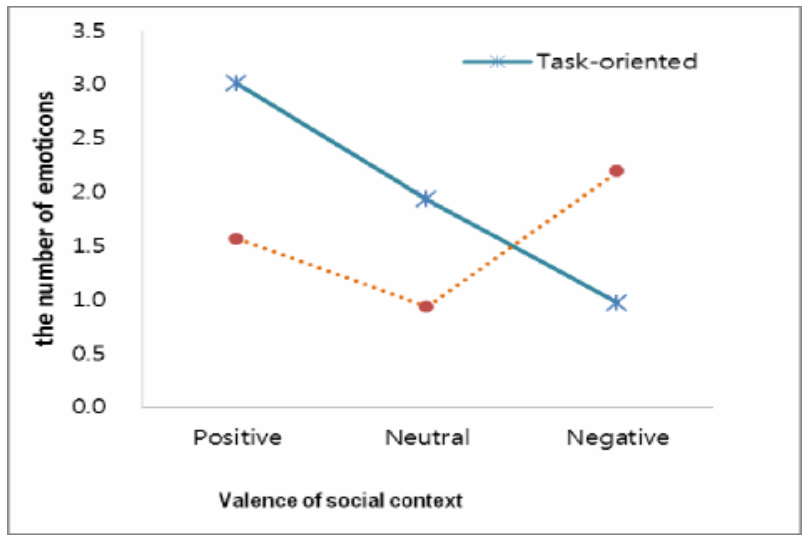

Figure 3: An interaction between kind of social context and valence

\section{DISCUSSION}

These results seemed in line with the social norms in face-to-face communication except $\mathrm{H} 1$. We present implications with a empirical experiment that the use of emoticons in social interaction on the mobile.

\section{REFERENCES}

[1] Rezabek, L. L., \& Cochenour, J. J. (1998). Visual cues in computer-mediated communication: Supplementing text with emoticons. Journal of Visual Literacy, 18, 201-215.

[2] Daft, R.L. and Lengel, R.H. (1984). Information richness: a new approach to managerial behavior and organizational design. Research in organizational behavior, 6, 191-233.

[3] Walther, J.B. (1992). Interpersonal effects in computer-mediated interaction: a relational perspective. Communication Research, 19, 52-90.

[4] Derks, D., Arjan E.R\& Grumbkow, von J., (2007). Emoticons and social interaction on the Internet: the importance of social context. Computers in Human Behavior, 23, 842-849.

[5] Ahn, W., \& Park, J (2010). Emoticon usage as an emotional sign in mobile. Proceeding of Korean Society of Design Science 2010, Seoul, South Korea.

[6] Lee, K., \& Jeon, S. (2005). New emoticon development and use in digital communication. Korean society of basic design \& art, 6, 307-315. 